\title{
Rho-Related GTP-Binding Protein RhoU
}

National Cancer Institute

\section{Source}

National Cancer Institute. Rho-Related GTP-Binding Protein RhoU. NCI Thesaurus. Code C114327.

Rho-related GT P-binding protein RhoU (258 aa, $28 \mathrm{kDa}$ ) is encoded by the human RHOU gene. This protein is involved in the regulation of cell migration. 